\title{
Diferencia regional de factores que afectan el desempeño escolar para la misma modalidad educativa
}

alma Sofía Santillán Hernández* y

JuAN Roberto VARgAS-SÁNChEz**

\section{RESUMEN}

En México existen diversas modalidades de enseñanza en la educación primaria. Las investigaciones sobre eficacia escolar identifican y cuantifican diferentes factores que influyen en el desempeño académico en cada una de éstas. En este trabajo se analiza el caso del estado de Hidalgo, México. Mediante pruebas estadísticas no paramétricas, se muestra la existencia de discrepancias en el logro escolar entre las regiones de la entidad para cada modalidad educativa. Además, con base en modelos econométricos de coeficientes beta, se explican las diferencias con los factores asociados a la composición de los estudiantes, a la escuela y a la localidad. Los resultados sugieren que hay modalidades educativas más sensibles que otras en cuanto a las interacciones entre el entorno socioeconómico regional y los factores escolares que afectan el logro escolar.

Palabras clave: rendimiento escolar, escuela primaria, análisis cuantitativo, modalidad educativa.

Clasificación JEL: C01, C51, I21.

* Profesora-investigadora en el área académica de Matemáticas y Física de la Universidad Autónoma del Estado de Hidalgo, México. ORCID ID: http://orcid.org/0000-0001-8736-8366. Correo electrónico: almasofia_santillan@uaeh.edu.mx

** Profesor-investigador en el área académica de Economía de la Universidad Autónoma del Estado de Hidalgo, México. ORCID ID: http://orcid.org/0000-0003-2817-3985. Correo electrónico: juanroberto_vargas@uaeh.edu.mx 


\section{ABSTRACT \\ Regional Difference of Factors Influencing School Performance for the Same Teaching Modality}

In México, there are different teaching modalities in primary education. Studies on school effectiveness identify and quantify various factors that influence on their academic performance. In this article the educational situation of Hidalgo State, Mexico is analyzed. Non-parametric statistical tests are performed to show the existence of discrepancies on school effectiveness between the different regions for each teaching modality. In addition, with econometric models of beta coefficient, these differences are explained by the factors associated with the composition of the students, schools and localities. The results suggest that some teaching modalities are more sensitive than others to the interactions between the regional socioeconomic context and the factors describing the student's environment.

Keywords: academic performance, primary schools, quantitative analysis, educational modality.

JEL Classification: C01, C51, I21

\section{INTRODUCCIÓN}

La educación edifica el acontecer de los seres humanos, esto es: dinamiza sus talentos y modifica su acceso a las oportunidades. Por lo tanto, educar supone construir sociedades más justas, incluyentes, prósperas y preparadas para enfrentar los retos de su permanencia. Las sociedades han puesto en los sistemas educativos la esperanza de un mejor porvenir mediante la adquisición de competencias que les permitan incorporarse a los sectores de mayor crecimiento y mejor proyección económica. Y es que, de acuerdo con Rose y Dyer (2008), la educación es un factor que ayuda tanto a reducir la pobreza como a romper con su herencia intergeneracional. No obstante, para desarrollar las capacidades individuales y conseguir las referidas competencias las escuelas tendrán que ser eficaces. Pero la incidencia de las escuelas tiene límites (Blanco, 2013). 
De acuerdo con Carvallo-Pontón (2010), los estudios sobre la eficacia escolar se originaron para identificar y cuantificar los factores que influyen en el desempeño académico. Para el caso mexicano, diversos autores han investigado los factores que afectan el logro escolar; no obstante, no consideran que cada modalidad educativa tiene particularidades que las hacen diferentes e incomparables entre sí. Precisamente por la marcada disparidad entre ellas, en este trabajo se analizan individualmente. Esta investigación abona a la literatura sobre la eficacia escolar desde una perspectiva regional, en particular al contexto de la escuela y al desempeño de los estudiantes de la misma modalidad educativa de las diferentes regiones de Hidalgo, México, estado localizado en el centro-oriente del país, donde habita $2.27 \%$ de la población del país.

Las seis modalidades educativas que aquí se consideran son: general unigrado; general multigrado; indígena unigrado; indígena multigrado; comunitaria, y privada. De acuerdo con los resultados de la prueba del Plan Nacional para la Evaluación de los Aprendizajes (Planea) para primaria, correspondientes a 2018, 22.7\% de los estudiantes hidalguenses de sexto grado de primaria se encuentran en el nivel de logro favorable en matemáticas, y es la indígena multigrado la modalidad que obtiene los resultados educativos más bajos, con apenas $13.6 \%$ de sus estudiantes en dicho nivel. Mientras que en la privada, $46 \%$ de los estudiantes cuentan con el nivel de logro favorable en matemáticas. La brecha en el logro escolar entre las modalidades educativas es más grande en el campo de conocimiento lenguaje y comunicación.

A finales de 2017, Hidalgo se regionalizó en cinco macro-regiones con base en la cercanía geográfica y similitud en las características de los municipios. La región IV tiene el menor porcentaje de estudiantes con nivel de logro favorable (18.8\%); mientras que la de mayor nivel es la I, con $29.4 \%$ de sus estudiantes en el nivel de logro favorable en matemáticas. Ante esta disparidad, el presente trabajo tiene dos objetivos: 1) aportar evidencia empírica sobre la existencia de diferencias estadísticas en el porcentaje promedio del alumnado que alcanza un nivel de logro académico favorable; las que se exhiben son entre regiones para cada modalidad educativa, y 2) explicar dichas desemejanzas mediante factores asociados a la composición de los estudiantes, a la escuela y a la localidad. De esta forma, se abona a la literatura especializada sobre las explicaciones de eficacia escolar diferenciada a nivel regional. 
El documento se encuentra organizado de la siguiente manera. En la primera sección se realiza una revisión de la literatura especializada en el tema. Posteriormente, se describen el método, los datos empleados y se presenta una caracterización de las regiones y modalidades educativas utilizadas para el análisis. Al final se discuten los resultados y se presenta la conclusión.

\section{REVISIÓN DE LA LITERATURA}

La investigación sobre eficacia escolar es prolífica y mantiene un vigoroso crecimiento. En la literatura especializada los conceptos de rendimiento y eficacia escolar no se utilizan como sinónimos. En general, el primero alude al nivel de dominio demostrado en un campo del conocimiento a partir de procesos de evaluación; su referencia es el estudiante. En el segundo, el nivel de observación es la escuela y, por tanto, los resultados académicos relevantes son los del agregado para distinguir las instituciones educativas eficaces. Al respecto, Murillo (2007) nos ofrece una panorámica sobre los elementos claves para que una escuela sea eficaz. Por su parte, Carvallo-Pontón (2010) ubica los antecedentes de la investigación sobre eficacia escolar en el trabajo de Coleman et al. (1966). De acuerdo con estos autores, el contexto de la escuela y el de la familia interactúan para que un estudiante obtenga buenos resultados escolares.

Diversos investigadores han encontrado que las características del vecindario en el que se habita, tales como el nivel de pobreza o la marginación, están asociadas con los resultados educativos de los niños, aún después de controlar por los antecedentes familiares (Ainsworth, 2002; Garner y Raudenbush, 1991; Gibbons, 2002). Lo anterior sugiere que la zona de residencia puede acentuar la desigualdad y limitar el acceso a oportunidades. En este sentido, DeLuca y Dayton (2009) realizan una revisión de estudios sobre la movilidad de la zona de residencia; los resultados muestran que los jóvenes que se mudaron de zonas pobres a vecindarios menos segregados tuvieron beneficios en sus resultados educativos en el corto plazo. Al respecto, Gibbons (2002) encuentra evidencia de que un niño que crece en un vecindario clasificado "con rezago" necesitaría padres con estudios universitarios para obtener 
las mismas oportunidades educativas que otro niño de un entorno promedio. ${ }^{1}$

En relación con el contexto familiar, el nivel socioeconómico es un elemento determinante del logro educativo (Sirin, 2005). El capital cultural es un canal mediante el cual se manifiesta el nivel socioeconómico. En México, Aguilar y Flores (2007) muestran evidencia de que incrementar una unidad de capital cultural ${ }^{2}$ aumenta 40 puntos a los resultados en una prueba de matemáticas aplicada a niños de sexto grado, y reportan que estos efectos son más grandes para Hidalgo. Por otro lado, González y Treviño (2018) investigan los factores familiares y escolares asociados al logro escolar de estudiantes de sexto grado de Nuevo León, México. Encuentran que el nivel de estudios de los padres es el que tiene mayor peso. Por su parte, Bravo et al. (2017) realizan un estudio para identificar las variables relevantes en el desempeño escolar de estudiantes chilenos de octavo grado de educación básica; descubren que las altas expectativas familiares son el factor diferenciador con mayor importancia en el rendimiento escolar. Mientras, Khattab (2015), usando datos de Inglaterra, precisa que los alumnos con altas aspiraciones de estudio tienden a obtener rendimientos académicos más altos. $^{3}$

Aunado a lo anterior, Cervini (2002) analiza los efectos del origen social del alumno y del contexto socioeconómico de la escuela y las provincias respecto al logro escolar en matemáticas y lengua para escuelas urbanas de educación primaria en Argentina. Con base en modelos lineales de niveles múltiples, encuentra que el contexto escolar es el mejor predictor del aprendizaje en ambas disciplinas.

Por su parte, Murillo (2008), utilizando datos de centros educativos en España, afirma que las escuelas de educación primaria con mayor rendimiento estudiantil cuentan, entre otras cosas, con la suficiencia y pertinencia de recursos materiales.

1 Un vecindario se clasifica con rezago si se encuentra en la parte inferior de la distribución de jerarquía educativa, es decir, con bajo porcentaje de adultos al menos con estudios universitarios.

2 El capital cultural se mide por medio de la escolaridad de los padres, sus expectativas académicas, el número de libros y acceso a internet en el hogar, así como la frecuencia de asistir al cine.

3 Las aspiraciones son medidas a partir de la pregunta realizada a los niños sobre sus planes para el momento en que tengan 16 y estén terminando el año de educación 11. 
A partir del nivel de marginación de las localidades en donde se encuentran las instituciones, Jiménez (2017) muestra la existencia de diferencias en el nivel de logro en lenguaje y comunicación de los estudiantes de sexto grado de educación básica de Baja California, México.

En distintos contextos se ha encontrado evidencia de que los estudiantes que trabajan tienen un menor rendimiento escolar (véase Neyt et al., 2019). En el caso específico de alumnos latinoamericanos de sexto grado de primaria, Murillo y Román (2014) demuestran que los niños que trabajan tienen en promedio entre 10 y 13 puntos menos en sus resultados de matemáticas y lectura, respectivamente, que aquellos que no trabajan fuera de su hogar.

En cuanto a las modalidades educativas, Hernández (2018) afirma que la prueba Planea 2015 evidencia que las escuelas multigrado son relativamente homogéneas en el rendimiento de sus alumnos. Por su parte, Backhoff et al. (2007) encuentran que los estudiantes de una escuela primaria indígena tienen más de un grado de retraso en su nivel de conocimientos en comparación con los de escuelas urbanas públicas; mientras que los estudiantes de escuelas privadas tienen un grado más que los de instituciones urbanas públicas. Sin embargo, estos efectos se reducen $80 \%$ al considerar ciertas características tanto de los estudiantes como de las escuelas. En este caso, la modalidad educativa explica más de $46 \%$ de la varianza observada entre los centros educativos.

La mayor disparidad entre las modalidades educativas ocurre entre la privada y la indígena. Por cada 16 alumnos de escuelas indígenas con bajo rendimiento académico, hay uno de escuela privada (Aguilar y Torreblanca, 2006). En cuanto a diferencias regionales, se ha encontrado evidencia de que las brechas entre modalidades en el logro educativo son más grandes que entre entidades federativas. De hecho, la disparidad entre entidades se explica por la diversa proporción de escuelas de cada modalidad educativa (INEE, 2005).

Asimismo, Blanco (2008) argumenta que el modelo de eficacia depende de las condiciones de operación de las escuelas; esto es, hay interacciones importantes entre el entorno sociocultural y los factores escolares, de ahí que no exista un único modelo de eficacia que aplique para todos. En este sentido, el presente trabajo proporciona evidencia acerca de que algunas modalidades educativas son más sensibles que otras a las relaciones entre las características socioeconómicas del entorno regional y los factores escolares que afectan la eficacia escolar; 
al menos esto se verifica a nivel regional en Hidalgo, como se mostrará en la sección de resultados.

\section{MÉTOdO}

Para verificar la existencia de diferencias en el porcentaje de estudiantes con nivel de logro favorable entre las macro-regiones, se utiliza la prueba no paramétrica de Kruskal-Wallis, la cual determina si alguna variable de interés es, en promedio, igual en tres o más grupos. Una de sus ventajas es que se requieren supuestos muy generales acerca del tipo de distribuciones de las cuales provienen las observaciones. Por ejemplo, la prueba sólo precisa que todas las observaciones sean independientes y que aquellas dentro de una muestra particular provengan de una sola población; también establece que las poblaciones analizadas tengan aproximadamente la misma forma (Kruskal y Wallis, 1952).

La prueba consiste en asignar un rango ${ }^{4}$ a cada observación y usar el siguiente estadístico

$$
H=\frac{12}{n(n+1)} \sum_{j=1}^{m} \frac{R_{j}^{2}}{n_{j}}-3(n+1)
$$

Donde $m$ son los grupos a analizar, en este caso $m=5, n_{\mathrm{j}}$ es el número de observaciones en la j-ésima muestra: $n=\sum_{\mathrm{j}=1}^{\mathrm{m}} n_{\mathrm{j}}$, y $R_{\mathrm{j}}$ es la suma de los rangos en la muestra $j$. Si cada muestra tiene más de cinco observaciones, entonces $\mathrm{H}$ se distribuye $\chi^{2}$ con $m-1$ grados de libertad.

Una vez identificadas las modalidades en las que existen diferencias entre regiones y con el fin de explicarlas, se realizan modelos econométricos de coeficientes beta, que nos permiten comparar la influencia relativa de cada una de las variables explicativas. De esta manera, para la estimación se deben estandarizar todos los factores incluidos en el modelo; es decir, a cada variable se le resta su media muestral y se divide por su desviación estándar muestral. Se utiliza el siguiente modelo:

4 Cada observación se ordena de menor a mayor; se asigna el rango 1 a la observación de menor magnitud; a la segunda mayor el 2, y así sucesivamente. 


$$
y_{i j}=\beta_{0}+\beta^{T} Z_{i j}+u_{i j}
$$

donde $y_{i j}$ es la estandarización del logaritmo del porcentaje de estudiantes con nivel de logro favorable en la escuela $i$ en la macro-región $j ; Z_{i j}$ es un vector de características estandarizadas de la escuela $i$ en la macro-región $j$, y $u_{i j}$ es el término de error, el cual se asume que tiene distribución normal con media cero y varianza constante. Se realiza este tipo de regresión para cada modalidad educativa. Los componentes del vector de coeficientes $\beta^{\mathrm{T}}$ de las variables estandarizadas $Z_{i j}$ son llamados coeficientes estandarizados o coeficientes beta. Esta modelación permitirá identificar y ordenar en cada región, según su importancia, los factores asociados a las diferencias esenciales entre las escuelas de la misma modalidad, para develar lo sustancial de lo aparente. Es decir, la variable asociada al coeficiente estandarizado con mayor valor absoluto será aquella que genere más cambio en desviaciones estándar en el logaritmo del porcentaje de estudiantes con nivel de logro favorable (en adelante, nos referiremos a él como logro escolar favorable).

\subsection{Datos y variables}

Los datos utilizados para este estudio provienen de los resultados de la prueba Planea 2018, aplicada a estudiantes de sexto grado de primaria del estado de Hidalgo (INEE, 2018). La prueba consiste en un examen de opción múltiple que tiene la finalidad de conocer el nivel de dominio de los aprendizajes clave del plan de estudios, los cuales son: insuficiente, apenas indispensable, satisfactorio y sobresaliente. De esta información se extrae la variable de interés en esta investigación: el porcentaje de estudiantes con nivel de logro favorable, la cual se construye agregando el porcentaje de estudiantes con niveles de logro satisfactorio y sobresaliente.

Con la finalidad de conocer información sobre el contexto en el que se desenvuelven los estudiantes y características de las escuelas, Planea aplica cuestionarios de contexto a los alumnos y directores. ${ }^{5}$

A partir de los cuestionarios aplicados a los alumnos se generan las siguientes variables agregadas por escuela:

Los cuestionarios de contexto también se aplican a padres de familia y docentes, pero en este estudio no se utiliza dicha información. 
- Índice socioeconómico promedio de los estudiantes (I. Socioeconómico). Para cada estudiante esta variable se genera con el promedio de las variables indicadoras de tenencia de luz eléctrica, agua potable, drenaje, piso firme, lavadora, refrigerador, horno de microondas, estufa, teléfono fijo, televisión de paga, computadora, televisión, automóvil, teléfono celular, tableta y disco óptico digital (DVD).

- Educación de la madre (Educación madre). Es el logaritmo del número de madres con educación al menos del nivel medio superior.

- Habla lengua indígena (Habla L. Indígena). Se refiere el logaritmo del número de estudiantes que hablan lengua indígena.

- Estudiantes que trabajan (Trabaja). Es el logaritmo del número de alumnos que trabajan en un negocio no familiar.

De los cuestionarios de contexto de los directores se genera la variable:

- Infraestructura escolar (Infr. Escolar). Se mide con el promedio de las variables indicadoras de existencia y suficiencia en la escuela del servicio de agua, baños, drenaje, energía eléctrica, aulas, biblioteca, sala de cómputo, laboratorios, mobiliario, pizarrones, material de lectura para uso de estudiantes, conexión a internet y computadora para uso de estudiantes.

También se hace uso del Cuestionario Formato 911 del ciclo escolar 2017-2018, que la Secretaría de Educación Pública (SEP) solicita a las escuelas al inicio y al final de cada ciclo escolar. De estas bases se construyen las siguientes variables:

- Alumnas inscritas (Alumnas). Se refiere al logaritmo del número de alumnas en la matrícula.

- Alumnos por docente (Alumnos por docente). Representa el logaritmo del número de alumnos por docente en la escuela.

La información del índice de marginación (iml) a nivel localidad del año 2010 proviene del Consejo Nacional de Población (Conapo). Todas las variables mencionadas se incluyen en el vector de variables $Z_{\mathrm{ij}}$ del modelo (2). La muestra de análisis consiste en las escuelas primarias del estado de Hidalgo que participaron en la aplicación de la 
prueba Planea 2018 y que contaron con información válida en cada una de sus fuentes. La muestra final consta de 2,791 escuelas, ubicadas en los 84 municipios del estado.

\subsection{Macro-regiones y modalidades educativas}

El 25 de diciembre del 2017 apareció en el Periódico Oficial del Estado de Hidalgo el decreto de la regionalización estatal en cinco macroregiones, cuyo propósito fue aprovechar las particularidades de cada zona y con ello impulsar el desarrollo regional. En la Figura 1 se muestran los municipios que conforman cada macro-región y su ubicación. En el Cuadro 1 se presentan algunas características socioeconómicas promedio de los municipios que conforman las macro-regiones.

En la macro-región I se encuentra la capital del estado, que se caracteriza por tener las mejores condiciones para el desarrollo humano y menores niveles de pobreza; además, cuenta con el mayor porcentaje

FIGURA 1

MUNICIPIOS QUE CONFORMAN LAS MACRO-REGIONES

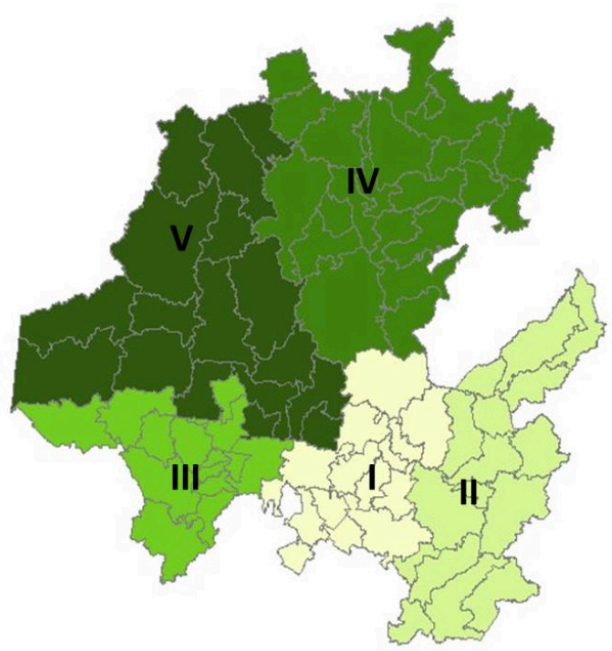

Fuente: elaboración propia.

Nota: en el Cuadro A.1 del apéndice se presentan los nombres de los municipios que conforman cada macroregión. 
promedio de estudiantes con el nivel de logro favorable. Por otro lado, en el norte del estado se ubica la macro-región IV, cuyo porcentaje promedio de pobres es de $77 \%$. El sector económico predominante es el primario y tiene el mayor porcentaje promedio de personas ocupadas que reciben menos de un salario mínimo como remuneración. ${ }^{6}$ Esta misma zona se caracteriza por los resultados más bajos en la prueba Planea, con un promedio de $10 \%$ de estudiantes con nivel favorable en lenguaje y comunicación. La macro-región III es la segunda con las mejores condiciones socioeconómicas. Mientras que las regiones II y $\mathrm{V}$ son similares en cuanto a desarrollo humano y pobreza, en materia educativa la región $\mathrm{V}$ tiene mejores resultados.

En México, las escuelas se clasifican en diferentes modalidades educativas: general multigrado, general unigrado, indígena multigrado, indígena unigrado, comunitaria y privada (ver cuadro 2).

\section{Cuadro 1}

CARACTERÍSTICAS SOCIOECONÓMICAS PROMEDIO DE LAS MACRO-REGIONES

\begin{tabular}{cccccc}
\hline (Índice de Desarrollo Humano (IDH) & I & II & III & IV & V \\
\hline \% en pobreza & 0.89 & 0.86 & 0.90 & 0.83 & 0.86 \\
\hline \% en pobreza extrema & 50.29 & 64.26 & 55.39 & 77.21 & 65.69 \\
\hline \% con carencia por rezago educativo & 8.32 & 18.46 & 9.75 & 30.76 & 16.51 \\
\hline \% sin escolaridad & 21.12 & 30.06 & 22.70 & 34.01 & 26.75 \\
\hline Años de escolaridad & 4.88 & 12.07 & 4.70 & 13.43 & 8.96 \\
\hline \% ocupados en sector primario & 8.83 & 7.60 & 8.60 & 6.87 & 7.78 \\
\hline \% ocupados en sector secundario & 8.25 & 22.02 & 13.79 & 44.64 & 28.05 \\
\hline \% ocupados en sector de servicios & 31.41 & 29.73 & 34.53 & 19.91 & 24.81 \\
\hline \% ocupados en sector de comercio & 42.99 & 30.96 & 34.30 & 25.10 & 32.05 \\
\hline \% con ingreso por trabajo de menos un salario mínimo & 16.02 & 15.92 & 15.76 & 9.12 & 13.59 \\
\hline \% con ingreso por trabajo entre 1 y 2 salarios mínimos & 31.94 & 33.91 & 28.62 & 31.29 & 33.92 \\
\hline \% con ingreso por trabajo de más de 2 salarios mínimos & 48.25 & 35.27 & 44.92 & 19.03 & 30.38 \\
\hline \% con nivel de logro favorable en matemáticas & 29.39 & 20.70 & 23.54 & 18.81 & 24.20 \\
\hline \% con nivel de logro favorable en lenguaje y comunicación & 26.04 & 14.52 & 20.13 & 10.12 & 16.56 \\
\hline Población total & 747,749 & 493,217 & 494,735 & 474,405 & 466,672 \\
\hline Total de municipios & 14 & 16 & 14 & 23 & 17 \\
\hline
\end{tabular}

Fuente: elaboración propia con base en datos del Programa de las Naciones Unidas para el Desarrollo 2015 para el IDH; CONEVAL 2010 (factor pobreza); Censo Económico 2013 (sector de ocupación); Prueba Planea de sexto grado 2018 (nivel de logro); Encuesta Intercensal 2015 (resto de las variables).

${ }^{1}$ Se refiere a personas de 15 años y más.

Nota: en el cuadro A.1 del apéndice se presentan los nombres de los municipios que conforman a cada macroregión.

6 Un salario mínimo diario equivale a 4.5 dólares estadounidenses del año 2015. 
Las escuelas multigrado están ubicadas principalmente en localidades rurales, en contextos de alta marginación; al menos un profesor imparte clases en dos o más grados en el mismo salón. Por su parte, las escuelas unigrado están ubicadas, en su mayoría, en localidades urbanas y cada profesor atiende a un grado escolar.

Cuadro 2

CARACTERÍSTICAS PROMEDIO DE LAS MODALIDADES EDUCATIVAS

\begin{tabular}{|c|c|c|c|c|c|c|}
\hline & General & & Indígena & & V & \\
\hline & Multigrado & Unigrado & Multigrado & Unigrado & Comunitaria & Privada \\
\hline Índice socioeconómico & 0.65 & 0.73 & 0.58 & 0.61 & 0.52 & 0.88 \\
\hline $\begin{array}{c}\% \text { madre al menos con } \\
\text { preparatoria }\end{array}$ & 12.80 & 30.76 & 9.20 & 12.75 & 6.76 & 80.64 \\
\hline$\%$ habla lengua indígena & 14.08 & 12.83 & 64.20 & 64.42 & 18.76 & 7.96 \\
\hline$\%$ trabaja & 47.86 & 39.48 & 57.21 & 56.50 & 44.39 & 25.60 \\
\hline Alumnos por docente & 17.17 & 23.68 & 13.43 & 16.70 & 9.20 & 16.76 \\
\hline$\%$ alumnas & 49.13 & 49.18 & 47.52 & 50.40 & 45.69 & 46.99 \\
\hline Índice de marginación & -0.33 & -0.96 & 0.04 & -0.03 & 0.10 & -1.29 \\
\hline Infraestructura escolar & 0.34 & 0.37 & 0.31 & 0.29 & 0.34 & 0.81 \\
\hline $\begin{array}{c}\% \text { nivel de logro favorable en } \\
\text { matemáticas }\end{array}$ & 20.03 & 26.30 & 13.58 & 14.07 & 19.06 & 45.26 \\
\hline $\begin{array}{c}\% \text { nivel de logro favorable en } \\
\text { lenguaje y comunicación }\end{array}$ & 12.40 & 18.44 & 7.02 & 7.68 & 13.82 & 46.08 \\
\hline Total de escuelas & 847 & 909 & 416 & 130 & 265 & 224 \\
\hline
\end{tabular}

Fuente: elaboración propia. La información de la infraestructura escolar proviene del cuestionario de contexto aplicado a los directores. La información sobre la matrícula de la escuela y la información sobre alumnos por docente se obtuvo del Cuestionario Formato 911 del ciclo escolar 2017-2018. La información sobre el nivel de logro proviene del reporte estatal de la prueba Planea 2018. El índice de marginación se obtuvo del Conapo. EI resto proviene del cuestionario de contexto aplicado a los estudiantes.

Respecto a las escuelas indígenas multigrado, se encuentran en localidades principalmente rurales donde reside población indígena; al menos un docente da clases a dos o más grados en el mismo salón. Las escuelas indígenas unigrado se ubican en localidades donde vive población indígena y cada docente atiende a un grado escolar. Los profesores ganan hasta 218 dólares estadounidenses mensuales menos que los profesores de escuelas generales (Delgado y Santos, 2015).

Con relación a las escuelas comunitarias, se ubican en localidades de menos de 100 habitantes; son atendidas por jóvenes recién egresados de secundaria o del nivel medio superior, a quienes se les brinda capa- 
citación para prestar el servicio. ${ }^{7}$ En esta modalidad educativa todas las escuelas son multigrado.

En cuanto a las instituciones privadas, que se sostienen con recursos propios, se encuentran primordialmente en localidades urbanas con bajos niveles de marginación. ${ }^{8}$ Sus estudiantes tienen las mejores condiciones familiares y escolares, así como los mejores resultados educativos.

Dentro de las escuelas públicas, la modalidad general unigrado tiene los mejores resultados educativos y los alumnos presentan las mejores condiciones económicas, aunque en este aspecto hay gran diferencia con la modalidad privada. Por otro lado, los estudiantes de las escuelas comunitarias son los que presentan las condiciones familiares más adversas y cuyo nivel de logro favorable en matemáticas es de $9 \%$ en promedio.

Los resultados educativos más bajos se encuentran en la modalidad indígena, cuya infraestructura es la más deficiente de todas las modalidades. En el informe "La educación para poblaciones en contextos vulnerables" (INEE, 2007), se realiza una comparación entre estudiantes de escuelas indígenas y de escuelas generales; los resultados muestran que la calidad del docente es la variable más importante para explicar el bajo rendimiento escolar de los alumnos de escuelas indígenas. Para igualar los resultados entre estas modalidades se necesita aumentar en 1.5 desviaciones estándar la calidad docente en las escuelas indígenas. ${ }^{9}$ Debido a esta marcada disparidad, se considera que estas modalidades no son comparables entre ellas, por lo que el análisis se realiza para cada modalidad.

7 Los jóvenes instructores de esta modalidad educativa prestan su servicio por 2 o 3 años y posteriormente reciben una beca para que puedan continuar con sus estudios.

8 La descripción de cada modalidad educativa se encuentra en https://www.inee.edu.mx/ evaluaciones/ecea/bases-de-datos-primaria-2014/

9 La calidad docente incluye puntualidad, asistencia a clases, tratar a los alumnos con respeto y ofrecer explicaciones claras para los estudiantes. 


\section{RESULTADOS}

La prueba de Kruskal-Wallis al 5\% de significancia muestra la existencia de diferencias entre macro-regiones en el promedio del porcentaje de estudiantes con nivel de logro favorable para el campo disciplinar de matemáticas en la modalidad educativa general multigrado. En el panel A de la Figura 2 se aprecia que las regiones II y III son las que presentan un menor nivel de logro favorable en matemáticas. No obstante, para lenguaje y comunicación no es posible rechazar la hipótesis nula de igualdad de medias entre macro-regiones. ${ }^{10}$

FIGURA 2

PORCENTAJE PROMEDIO DE ESTUdIANTES EN EL NIVEL DE LOGRO ESCOLAR FAVORABLE EN MATEMÁTICAS (POR MODALIDAD EDUCATIVA Y MACRO-REGIÓN)

(A) General

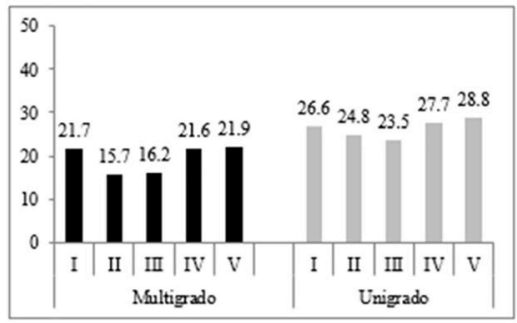

(C) Comunitaria

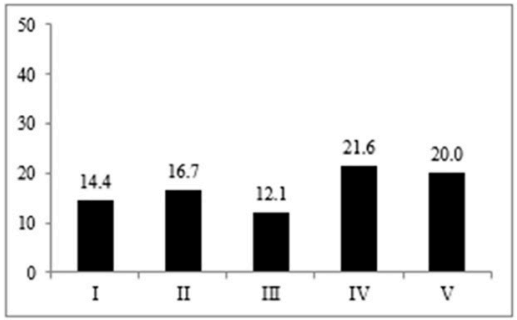

(B) Indigena

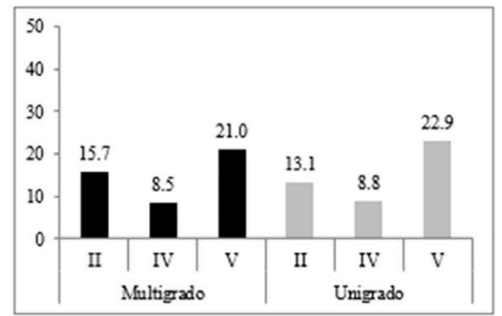

(D) Privada

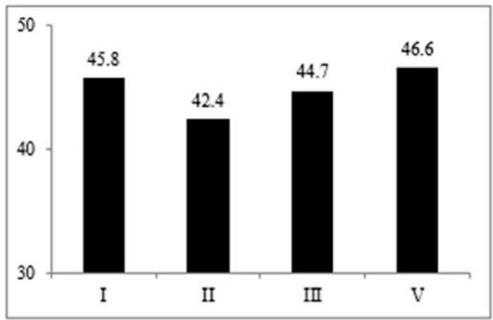

Fuente: elaboración propia con base en la prueba Planea 2018. Nota: la categoría logro escolar favorable incluye los niveles de logro satisfactorio y sobresaliente.

10 En el cuadro A.2 del apéndice se registran los resultados del estadístico H y los valores p de las pruebas de hipótesis de igualdad de medias en el porcentaje de estudiantes con nivel de logro favorable entre macro-regiones. 
En el caso de la modalidad general unigrado, al 10\% de significancia, se encuentra diferencia de medias entre macro-regiones para matemáticas, y al 1\%, para lenguaje y comunicación. El panel A de las figuras 2 y 3 revela que las escuelas de la región IV tienen el menor porcentaje de niños con nivel de logro favorable en lenguaje y comunicación; no obstante, es la segunda mejor región en logro favorable en matemáticas. Por su parte, en las escuelas indígenas unigrado y multigrado existen diferencias, al $1 \%$ de significancia, tanto para lengua y comunicación como para matemáticas.

FIGURA 3

PORCENTAJE PROMEDIO DE ESTUDIANTES EN EL NIVEL DE LOGRO ESCOLAR FAVORABLE EN LENGUAJE Y COMUNICACIÓN (POR MODALIDAD EDUCATIVA Y MACRO-REGIÓN)

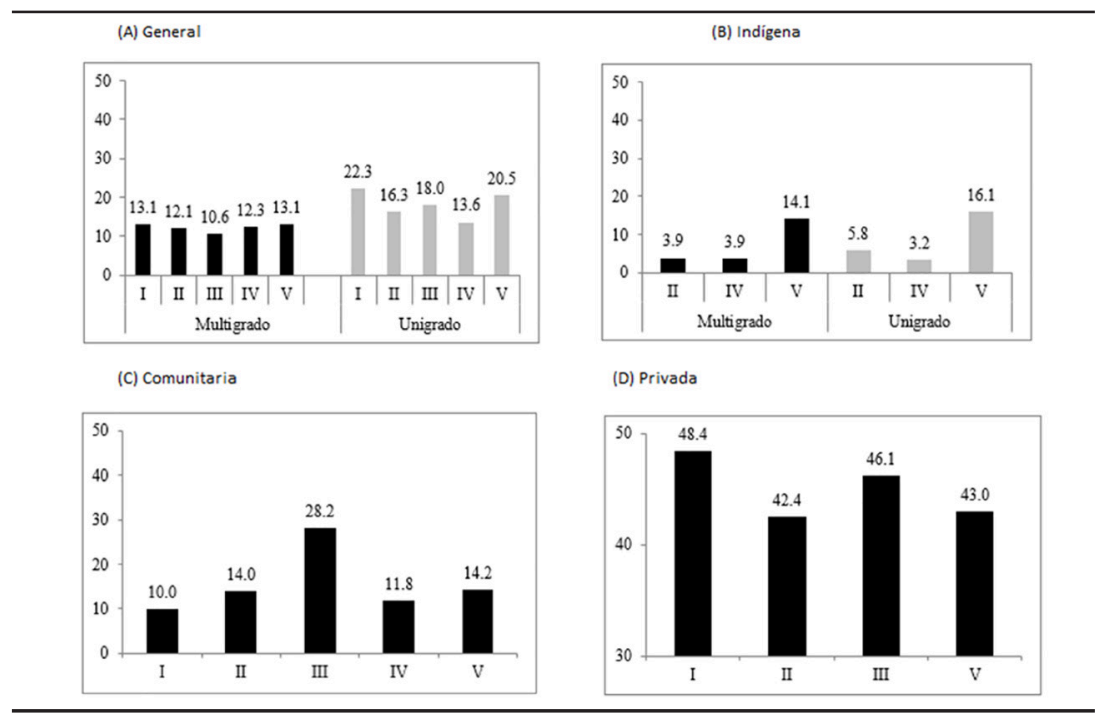

Fuente: elaboración propia con base en la prueba Planea 2018. Nota: la categoría logro escolar favorable incluye los niveles de logro satisfactorio y sobresaliente.

En el panel B de las figuras 2 y 3 se aprecia que, para la modalidad de escuelas indígenas, la región $\mathrm{V}$ muestra el mayor porcentaje de estudiantes con nivel de logro favorable tanto en matemáticas como en lenguaje y comunicación. Aunado a ello, en las escuelas privadas y en las comunitarias no hay evidencia estadística de diferencias entre las macro-regiones. En el panel D de las figuras 2 y 3, se observa que las escuelas privadas tienen los mayores porcentajes de estudiantes en nivel 
de logro favorable tanto en matemáticas como en lenguaje y comunicación en todas las macro-regiones, relativo al resto de las modalidades.

\subsection{Factores asociados a las diferencias macro-regionales y a la modalidad educativa}

Para explicar las diferencias encontradas, se realizaron modelos econométricos para cada macro-región y modalidad educativa. Las variables independientes significativas asociadas al logro escolar favorable se identificaron y ordenaron de acuerdo a su coeficiente beta. En el Cuadro 3 se muestran los resultados de las estimaciones del modelo (2), pero sólo se presentan las variables significativas con mayor peso dentro de cada región y modalidad educativa. De esta forma, los resultados expuestos en dicho cuadro muestran que en cada modalidad educativa y región hay diferentes factores asociados al logro escolar favorable. Cabe aclarar que cuando se reportan varios coeficientes como los de mayor peso, se debe a que mediante pruebas $\mathrm{F}$ no es posible rechazar la hipótesis de igualdad de coeficientes. ${ }^{11}$ La lectura se debe hacer con la consideración de que se trata de resultados en el promedio y manteniendo lo demás constante. Por ejemplo, en la modalidad general multigrado, para la región I y la asignatura de matemáticas, la variable estudiantes que trabajan es la de mayor peso asociada al logro escolar favorable; así, el incremento de una desviación estándar de esta variable disminuye el logro escolar favorable en 0.389 desviaciones estándar.

Siguiendo con la modalidad general multigrado y matemáticas, en la región IV, las variables hablar lengua indígena y estudiantes que trabajan están ligadas de manera negativa con el logro escolar favorable. En particular, el incremento de una desviación estándar de cada una de ellas disminuye 0.206 desviaciones estándar el logro escolar. Por su parte, en las regiones II y V, la variable estudiantes que trabajan es la de mayor peso asociada al logro escolar favorable.

11 En el cuadro A.3 del apéndice se muestran los estadísticos F y los valores p de las pruebas de hipótesis de igualdad de coeficientes. 


\section{CUADRO 3}

VARIABLES DEL MODELO (2) CON MAYOR COEFICIENTE BETA (POR MODALIDAD EDUCATIVA Y CAMPO DISCIPLINAR)

\begin{tabular}{|c|c|c|c|c|c|}
\hline & 1 & II & III & IV & V \\
\hline \multicolumn{6}{|c|}{ General multigrado } \\
\hline Matemáticas & Trabaja $(-0.389 * *)$ & Trabaja $\left(-0.457^{* * *}\right)$ & NS & $\begin{array}{l}\text { Habla L. Indígena } \\
\left(-0.206^{* * *}\right), \\
\text { Trabaja }\left(-0.206^{* * *}\right)\end{array}$ & $\begin{array}{c}\text { Trabaja } \\
\left(-0.561^{* * *}\right)\end{array}$ \\
\hline \multicolumn{6}{|c|}{ General unigrado } \\
\hline Matemáticas & $\begin{array}{l}\text { Eduación madre } \\
\left(0.347^{* * *}\right) \\
\text { I. Socioeconómico } \\
(0.254)\end{array}$ & $\begin{array}{l}\text { Eduación madre } \\
\left(0.376^{* * *}\right) \\
\text { I. Socioeconómico } \\
\left(0.206^{* *}\right)\end{array}$ & NN & Infr. Escolar $\left(0.131^{*}\right)$ & $\begin{array}{c}\text { I. } \\
\text { Socioeconómico } \\
\left(0.224^{* * *}\right)\end{array}$ \\
\hline Lenguaje & $\begin{array}{l}\text { Educación madre } \\
\qquad\left(0.425^{* *}\right)\end{array}$ & $\begin{array}{l}\text { Educación madre } \\
\qquad\left(0.303^{* *}\right)\end{array}$ & NN & $\begin{array}{l}\text { Educación madre } \\
\qquad\left(0.276^{* *}\right)\end{array}$ & $\begin{array}{c}\text { Educación madre } \\
\qquad\left(0.271^{* *}\right)\end{array}$ \\
\hline \multicolumn{6}{|c|}{ Indígena multigrado } \\
\hline Matemáticas & $N E$ & $\begin{array}{c}\text { Alumnos por } \\
\text { docente }\left(-0.66^{* * *}\right)\end{array}$ & NE & NN & Trabaja $\left(-0.323^{*}\right)$ \\
\hline Lenguaje & NE & Trabaja $\left(-0.713^{* *}\right)$ & NE & $\begin{array}{c}\text { Trabaja }\left(-0.433^{*}\right) \text {, } \\
\text { Habla L. Indigena } \\
\left(-0.37^{*}\right)\end{array}$ & $\begin{array}{c}\text { Trabaja } \\
\left(-0.541^{* * *}\right)\end{array}$ \\
\hline \multicolumn{6}{|c|}{ Indígena unigrado } \\
\hline Matemáticas & NE & $\begin{array}{l}\text { I. Socioeconómico } \\
\left(0.848^{* * *}\right)\end{array}$ & NE & $\begin{array}{l}\text { Alumnos por docente } \\
\qquad\left(-0.346^{*}\right)\end{array}$ & $\begin{array}{c}\text { Trabaja } \\
\left(-0.395^{* *}\right)\end{array}$ \\
\hline Lenguaje & NE & Trabaja $(-0.606 * *)$ & NE & Trabaja $(-0.749 * * *)$ & Trabaja $\left(-0.45^{*}\right)$ \\
\hline
\end{tabular}

Fuente: estimaciones propias. Coeficiente beta significativo al *10\%, **5\%, ***1\%. En todos los casos reportados, los términos de error cumplen el supuesto de normalidad y los errores estándar son robustos a heterocedasticidad. Cuando no hay normalidad en los términos de error del modelo, la celda se distingue con (NN); una región sin escuelas se reporta con (NE), y cuando no hay significancia estadística en los coeficientes estimados con (NS). Por razones de espacio, en el cuadro A.4 del apéndice sólo se exhiben los resultados de las pruebas de normalidad realizadas a los términos de error del modelo correspondiente a las escuelas indígenas unigrado. No obstante, los resultados de todas las pruebas de normalidad realizadas a todos los modelos están a disposición de los lectores que las soliciten.

Destaca que en la modalidad general unigrado la educación de la madre es la variable preponderante. En el caso de matemáticas, si se mantiene todo lo demás constante, el aumento de una desviación estándar de la citada variable incrementa en 0.347 y 0.376 desviaciones estándar el logro escolar favorable en las regiones I y II, respectivamente. Para el campo disciplinar de lenguaje y comunicación, el incremento es de $0.425,0.303,0.276$ y 0.271 , en las regiones I, II, IV y V, respectivamente. En la región I, la variable índice socioeconómico es estadísticamente similar a la variable educación de la madre, por ello se reportan como las de mayor peso en esta región; la primera citada se asocia posi- 
tivamente con el logro académico favorable. En la región II son dos las variables más relevantes: la mencionada educación de la madre y el índice socioeconómico; ambas se asocian positivamente con el logro escolar favorable.

En el caso de la modalidad indígena multigrado para matemáticas, en la región II, la variable con mayor magnitud es el número de alumnos por docente; mientras que en la región $\mathrm{V}$ es la variable estudiantes que trabajan; ambas se asocian de forma negativa con el logro escolar favorable. Para lenguaje y comunicación, la de mayor relevancia en las regiones II, IV y V es la variable estudiantes que trabajan, aunque en la región IV, también la variable habla lengua indígena está relacionada de forma negativa con el logro escolar.

Finalmente, en la modalidad educativa indígena unigrado, en lenguaje y comunicación, la variable estudiantes que trabajan es la de mayor relevancia y está asociada de forma negativa con el logro escolar favorable en las regiones II, IV y V. Para matemáticas, en la región II el índice socioeconómico se vincula positivamente, y en la región IV, la variable alumnos por docente se relaciona de forma negativa. En la región $\mathrm{V}$, la variable estudiantes que trabajan es la relevante. De esta manera, manteniendo lo demás constante, el incremento de una desviación estándar de tal variable reduce en 0.395 desviaciones estándar el logro escolar favorable.

\section{CONCLUSIONES}

Los resultados de la investigación muestran que no hay diferencias regionales en el porcentaje de estudiantes con nivel de logro escolar favorable en las modalidades educativas privada y comunitaria. Lo anterior significa que, en promedio, el porcentaje de niñas y niños con nivel de logro favorable de una escuela privada o comunitaria, de la zona con mayor desarrollo económico de la entidad, por ejemplo, del área metropolitana del sur del estado, es similar al de una escuela privada o comunitaria de la zona con mayor rezago, por ejemplo, el norte del estado. Lo análogo para escuelas indígenas y escuelas generales no se sostiene. Este resultado es relevante porque aporta evidencia empírica para sostener la hipótesis de que las interacciones entre el entorno socioeconómico de los centros educativos y los factores escolares que inciden en el logro escolar influyen de forma diferenciada en los tipos de modalidad educativa. 
Probablemente lo más distintivo de esta investigación es hacer evidente la clara influencia de factores específicos en el logro escolar para distintos contextos, ya que las diferencias estadísticas identificadas en las modalidades general e indígena se explican porque las variables con mayor peso asociadas al logro escolar son particulares de cada región, modalidad educativa y campo del conocimiento. Por ejemplo, las escuelas de la modalidad general unigrado se ubican principalmente en zonas urbanas y con relativo mayor nivel de desarrollo económico; aquí, la variable de mayor incidencia en el logro escolar es la educación de la madre. Pero en la modalidad indígena, lo que más influye de forma negativa es el hecho de que las niñas y los niños tengan que trabajar. Entonces, la educación de la madre y el trabajo infantil son factores relevantes en la explicación de las divergencias regionales en cada modalidad. Ambos efectos resultan ser consistentes con la literatura. Concretamente, González y Treviño (2018) afirman que un mayor nivel de educación de los padres genera un ambiente familiar que propicia el desarrollo de las habilidades cognitivas de los hijos. Respecto al trabajo infantil, los resultados encontrados son congruentes con otras investigaciones: el hecho de que los niños y las niñas trabajen tiene efectos negativos en el logro escolar (Neyt et al., 2019).

El estudio muestra que hablar lengua indígena es otra variable relevante para algunas modalidades y regiones. De acuerdo con Noé et al. (2005), si las aulas con población indígena fueran más homogéneas étnicamente, se mejoraría el logro escolar tanto de los indígenas como de los no indígenas. En el mismo tenor, Blanco (2017) muestra que las brechas de aprendizaje entre los niños indígenas y no indígenas se explican principalmente por los factores socioculturales y en menor proporción por las expectativas educativas y la modalidad escolar; el autor asevera que los problemas de educabilidad de los indígenas son similares a los estudiantes no indígenas que viven en situaciones socioeconómicas semejantes. En esta investigación se muestra que en algunas macro-regiones se sostiene esta afirmación, pero no en todas.

Finalmente, aquí se evidencia que las condiciones institucionales y estructurales no son neutrales respecto al proceso educativo. Los vínculos entre el entorno socioeconómico y las modalidades educativas son relevantes y se deben considerar a la hora de diseñar políticas eficaces de calidad educativa. 


\section{Bibliografía}

Aguilar, Miguel y Omar Torreblanca (eds.) (2006). El aprendizaje del español y las matemáticas en la educación básica en México: sexto de primaria y tercero de secundaria. Instituto Nacional para la Evaluación de la Educación, México.

Aguilar, Miguel y Diana Luz Flores (eds.) (2007). Aprendizaje y desigualdad social en México: implicaciones de política educativa en el nivel básico. Instituto Nacional para la Evaluación de la Educación, México.

Ainsworth, James (2002). "Why does it take a village? The mediation of neighborhood effects on educational achievement". Social Forces 81(1), 117-152, https://doi.org/10.1353/sof.2002.0038

Backhoff, Eduardo, Arturo Bouzas, Carolina Contreras, Eduardo Hernández y Marisela García (2007). Factores escolares y aprendizaje en México. El caso de la educación básica. México. Instituto Nacional para la Evaluación de la Educación, México.

Blanco, Emilio (2017). "Los alumnos indígenas en México: Siete hipótesis sobre el rezago en los aprendizajes de nivel primario". REICE: Revista Iberoamericana sobre Calidad, Eficacia y Cambio en Educación, 15(3), 81-112.

Blanco, Emilio (2013). Los límites de la escuela. Educación, desigualdad y aprendizajes en México. El Colegio de México, México.

Blanco, Emilio (2008). "Factores escolares asociados a los aprendizajes en la educación primaria mexicana: un análisis multinivel". REICE. Revista Iberoamericana sobre Calidad, Eficacia y Cambio en Educación, 6(1), 58-84.

Bravo, Mónica, Sonia Salvo, Manuel Mieres, Juan Mansilla y Christian Hederich (2017). "Perfiles de desempeño académico: la importancia de las expectativas familiares". Perfiles latinoamericanos, 25(50), 361-386, http:// dx.doi.org/10.18504/p12550-016-2017.

Carvallo-Pontón, Mauricio (2010). "Eficacia escolar: antecedentes, hallazgos y futuro”. Magis, Revista Internacional de Investigación en Educación, 3(5), 199-214.

Cervini, Rubén (2002). "Desigualdades en el logro académico y reproducción cultural en Argentina". Revista mexicana de investigación educativa, 7(16), 445-500. México.

Coleman, James, Ernest Q. Campbell, Carol J. Hobson, James McPartland, Alexander M. Mood, Frederic D. Weinfeld, y Robert, L. York (1966). Equality of educational opportunity. Washington, DC: Government Printing Office, EUA. 
Delgado Alejandra y Annete Santos (coords.). (2015). Los docentes en México. Informe 2015. Instituto Nacional para la Evaluación de la Educación, SEP, México.

DeLuca, Stefanie y Elizabeth Dayton (2009). "Switching social contexts: The effects of housing mobility and school choice programs on youth outcomes". Annual Review of Sociology, 35, 457-491, https://doi.org/10.1146/ annurev-soc-070308-120032.

Garner, Catherine y Stephen Raudenbush (1991). "Neighborhood effects on educational attainment: A multilevel analysis". Sociology of education, 64(4), 251-262. https://doi.org/10.2307/2112706.

Gibbons, Steve (2002). "Neighborhood effects on educational achievement". CCE Discussion Papers 0018. Centre for the Economics of Education, London School of Economics, Inglaterra.

González, Mario y Diana Treviño (2018). "Logro educativo y factores asociados en estudiantes de sexto grado de educación primaria en el estado de Nuevo León, México". Perfiles educativos, 40(159), 107-125.

Hernández, Eduardo (2018). "El Aprendizaje en Escuelas Multigrado Mexicanas en la Prueba Planea". REICE: Revista Electrónica Iberoamericana sobre Calidad, Eficacia y Cambio en Educación, 16(3), 123-138. https:// doi.org/10.15366/reice2018.16.3.007.

INEE (Instituto Nacional para la Evaluación de la Educación) (2018). "Planea, Resultados nacionales 2018. Sexto de primaria”. México.

INEE (2007). "La educación para poblaciones en contextos vulnerables". Informe Anual 2007, México.

INEE (2005). “Resultados de logro educativo". Factores que los explican. México.

Jiménez, José Alfonso (2017). “Una mirada hacia la calidad de la educación primaria en Baja California, México: marginación escolar y equidad en sus resultados". REICE: Revista Iberoamericana sobre Calidad, Eficacia y Cambio en Educación, 15(3), 35-48.

Khattab, Nabil (2015). "Student's aspirations, expectations and school achievement: what really matters?". British Educational Research Journal 41(5), 731-748. https://doi.org/10.1002/berj.3171.

Kruskal, William y Allen Wallis (1952). "Use of ranks in one-criterion variance analysis". Journal of the American Statistical Association, 47(260), 583-621.

Murillo, Francisco Javier (2008). "Hacia un modelo de eficacia escolar. Estudio multinivel sobre los factores de eficacia en las escuelas españolas". REICE. Revista Iberoamericana sobre Calidad, Eficacia y Cambio en Educación, 6(1), 4-28.

Murillo, Francisco Javier (coord.) (2007). Investigación iberoamericana sobre eficacia escolar. Convenio Andrés Bello, Bogotá. 
Murillo, Francisco Javier y Marcela Román (2014). “Consecuencias del trabajo infantil en el desempeño escolar: Estudiantes latinoamericanos de educación primaria". Latin American Research Review, 49(2), 84-106.

Neyt, B., E. Omey, Verhaest y S. Baert (2019). "Does student work really affect educational outcomes? A review of the literature". Journal of Economic Surveys, 33(3), 896-921. https://doi.org/10.1111/joes.12301

Noe, David, Jorge Rodríguez e Isabel Zúñiga (2005). "Brecha étnica y influencia de los pares en el desempeño escolar: Evidencia para Chile”. Serie Políticas Sociales (102). División de Desarrollo Social, CEPAL, Santiago de Chile.

Rose, Pauline y Caroline Dyer (2008). "Chronic poverty and education: A review of literature”. Chronic Poverty Research Centre Working Paper 131. http://dx.doi.org/10.2139/ssrn.1537105.

Sirin, Selcuk (2005). "Socioeconomic status and academic achievement: A meta-analytic review of research". Review of educational research, 75(3), 417-453. https://doi.org/10.3102/00346543075003417. 


\section{ANEXO}

CUADRO A.1

MUNICIPIOS QUE CONFORMAN CADA MACRO-REGIÓN

\begin{tabular}{|c|c|}
\hline $\begin{array}{l}\text { Macro- } \\
\text { región }\end{array}$ & Municipios \\
\hline 1 & $\begin{array}{c}\text { Atotonilco el Grande, Epazoyucan, Huasca, Mineral del Chico, Mineral del Monte, Omitlán, Pachuca, } \\
\text { Mineral de la Reforma, San Agustín Tlaxiaca, Villa de Tezontepec, Tizayuca, Tolcayuca, Zapotlán y } \\
\text { Zempoala. }\end{array}$ \\
\hline II & $\begin{array}{c}\text { Acatlán, Acaxochitlán, Agua Blanca, Almoloya, Apan, Cuautepec, Emiliano Zapata, Huehuetla, Metepec, } \\
\text { San Bartolo Tutotepec, Santiago Tulantepec, Singuilucan, Tenango de Doria, Tepeapulco, Tlanalapa y } \\
\text { Tulancingo. }\end{array}$ \\
\hline
\end{tabular}

III Ajacuba, Atitalaquia, Atotonilco de Tula, Chapantongo, Mixquiahuala, Nopala, Progreso, Tepeji del Río, Tepetitlán, Tetepango, Tezontepec de Aldama, Tlahuelilpan, Tlaxcoapan y Tula.

Atlapexco, Calnali, Chapulhuacán, Eloxochitlán, Huautla, Huazalingo, Huejutla, Jaltocán, Juárez, Lolotla,
San Agustín Metzquititlán, Metztitlán, Molango, San Felipe Orizatlán, Pisaflores, Tepehuacán de
Guerrero, Tianguistengo, Tlahuiltepa, Tlanchinol, Xochiatipan, Xochicoatlán, Yahualica y Zacualtipán.
Actopan, Alfajayucan, El Arenal, Cardonal, Chilcuautla, Francisco I. Madero, Huichapan, Ixmiquilpan,
Jacala, La Misión, Nicolás Flores, Pacula, San Salvador, Santiago de Anaya, Tasquillo, Tecozautla y
Zimapán.

Fuente: elaboración propia con base en el Periódico Oficial del del Estado de Hidalgo, 25 de diciembre de 2017.

\section{CUADRO A.2}

RESULTADOS DE LAS PRUEBAS DE DIFERENCIA DE MEDIAS EN EL PORCENTAJE DE ESTUDIANTES CON NIVEL DE LOGRO FAVORABLE ENTRE MACRO-REGIONES

\begin{tabular}{|c|c|c|c|c|c|}
\hline & & \multicolumn{2}{|c|}{ Matemáticas } & \multicolumn{2}{|c|}{ Lenguaje y comunicación } \\
\hline \multicolumn{2}{|c|}{ Modalidad } & $\mathrm{H}$ & Valor $\mathrm{p}$ & $\mathrm{H}$ & Valor $p$ \\
\hline \multirow[t]{2}{*}{ General } & Multigrado & 16.13 & 0.0300 & 4.87 & 0.3012 \\
\hline & Unigrado & 8.98 & 0.0616 & 38.23 & 0.0001 \\
\hline \multirow[t]{2}{*}{ Indígena } & Multigrado & 12.18 & 0.0068 & 14.81 & 0.0020 \\
\hline & Unigrado & 16.71 & 0.0002 & 22.96 & 0.0001 \\
\hline Comunitaria & & 2.95 & 0.5658 & 3.02 & 0.5547 \\
\hline Privada & & 4.27 & 0.3703 & 4.48 & 0.3454 \\
\hline
\end{tabular}

Fuente: elaboración propia. Estimaciones realizadas usando el programa STATA 14. Nota: $\mathrm{H}$ refiere al estadístico de la prueba Kruskal-Wallis. 


\section{CUADRO A.3}

RESULTADOS DE LA PRUEBA DE HIPÓTESIS DE IGUALDAD ENTRE LOS COEFICIENTES DE MAYOR MAGNITUD DEL MODELO (2)

\begin{tabular}{ccccc}
\hline Modalidad & Asignatura & Macro-región & F & Valor p \\
General multigrado & Matemáticas & IV & 0.0400 & 0.8437 \\
General unigrado & Matemáticas & I & 2.3600 & 0.1258 \\
General unigrado & Matemáticas & II & 2.9800 & 0.0866 \\
\hline Indígena multigrado & Lenguaje & IV & 0.0300 & 0.8659 \\
\hline
\end{tabular}

Fuente: elaboración propia.

Nota: $\mathrm{F}$ se refiere al estadístico de la prueba de igualdad de coeficientes.

CUADRO A.4

VALOR P DE LAS PRUEBAS DE NORMALIDAD DE LOS TÉRMINOS DE ERROR DE LA ECUACIÓN (2) PARA LA MODALIDAD INDÍGENA UNIGRADO

\begin{tabular}{|c|c|c|c|}
\hline \multicolumn{4}{|c|}{ Macro-región } \\
\hline & II & IV & V \\
\hline \multicolumn{4}{|c|}{ Matemáticas } \\
\hline SK & 0.256 & 0.057 & 0.664 \\
\hline SW & 0.079 & 0.278 & 0.834 \\
\hline SF & 0.115 & 0.154 & 0.859 \\
\hline$J B$ & 0.351 & 0.098 & 0.663 \\
\hline \multicolumn{4}{|c|}{ Lenguaje y comunicación } \\
\hline SK & 0.216 & 0.032 & 0.349 \\
\hline SW & 0.199 & 0.098 & 0.369 \\
\hline SF & 0.204 & 0.059 & 0.393 \\
\hline$J B$ & 0.430 & 0.062 & 0.469 \\
\hline
\end{tabular}

Fuente: elaboración propia.

Nota: SK representa la prueba de curtosis y asimetría. SW indica la prueba Shapiro Wilk. SF es la prueba Shapiro-Francia. JB indica la prueba Jarque Bera. 